\title{
Design and Implementation of Human Body Shape Analysis System Based on Electronic Tailored Clothing eMTM
}

\author{
Zhenhua Wen \\ \{wenzhenhuawh@163.com\} \\ Wuhan Polytechnic wuhan 430074, China
}

\begin{abstract}
Body shape analysis, as a key technology in the production of eMTM, plays a very important role in the production of eMTM. The results of shape analysis will directly influence the design of clothing prototype and the generation of garment pattern. Accurate body shape analysis is an important guarantee for the customer to meet the requirement of garment fit. Therefore in our country started for eMTM body shape analysis research, the establishment of efficient and accurate body shape analysis system, will actively guide and help the research and extension of eMTM production in China and China's garment enterprises digital development. In this paper, the human body size analysis of eMTM is studied, and the production is described in detail, and the aim of the research is to establish the human body size analysis system based on eMTM. The paper analyzes the defect of the traditional shape analysis method, presents the multivariate statistical analysis methods introduced ergonomic research field. By the means of clustering analysis of multivariate statistical analysis on the target group human body feature analysis, and the selection of feature variables in traditional shape analysis methods are improved, the shape analysis results can more accurately reflect the human body characteristics. At the same time, this paper proposes the use of advanced J2EE application architecture, the establishment of a Web based human body analysis system, in an efficient system platform to achieve a variety of human body shape analysis.
\end{abstract}

Keywords: Electronic tailored clothing; 3D body scanning; body shape analysis; J2EE; cluster analysis

\section{Introduction}

Clothing is a silent expression of personality, and the body of clothing is the best tool to show the advantages of individual figure. With the continuous development of society and economy. In this customer oriented consumer era, people began to pursue personalized garment design, hope to be able to wear out meet their shape features and aesthetic needs, for their own tailor-made clothing. Therefore, clothing production from the past in large quantities, less variety toward small batches, multi variety and even the direction of the development of tailor-made. At present in Europe and the United States [1], tailored clothing production accounted for the market share, and will become the future development trend of clothing production. With the development of modern computer technology and network technology, many foreign countries has launched a electronic tailored clothing one of eMTM 
research and the use of digital, networked method to achieve fast and efficient tailored clothing production. In recent years, with China's garment enterprises and foreign exchange continues to close, clothing enterprises in our country have realized the importance of electronic tailored clothing research, and hope in our country to carry out research of eMTM production, to closely follow the development trend of the international apparel production, accelerate the process of digital development of China's garment enterprises. The fit of clothing occupies a very important position in the fashion design. For consumers, the size of the wrong, the size of different, and then satisfied with the clothing, but also to give up. For manufacturers, the sales area of clothing fit and matching is not good lead to a large backlog of clothing. 2013, due to the nature of the problem, a total of 100 million shirts in china. Using the correct method to analyze the user's body size, to accurately judge the user's body size, is the key to solve the problem of clothing fit. Therefore, the shape analysis is a key link in eMTM production, accurate result of shape analysis will greatly improve the accuracy of garment pattern design to meet user requirements for garment fitting and matching. This handmade method has been in the world for many years, and we have said that the "tailor" is engaged in the work of handmade custom clothing. However, there are some shortcomings of the manual custom clothing, the first is the consumption of a long time. Second clothing design is based on the experience and judgment of the tailor, the lack of quantitative standards, is not conducive to the production of large quantities of clothing, but also not conducive to the use of customized clothing for different places. Third is the design of the accuracy of the problem, need to be amended. The second type of custom tailored to the series of apparel sizes and styles of standard, first determine the clothing sizes corresponding to the user type, according to the custom in garment sizes. The time required for this customization is relatively small, but also conducive to the production of large quantities of clothing [2].

\section{Analysis of the Key Technologies Involved in the System}

Flex is the Flex Adobe, which is a free open source framework for efficient open source code, and is often used to build highly expressive Web applications. These Web applications through the use of Flash Player Adobe and AIR Adobe tools, you can in a number of browser platforms, operating platform on the use of cross. Flex applications can only through the flex framework to develop, but Adobe Flash Builder "software can through the use of intelligent coding and other functions to speed up the process of software development. The use of Flex to create RIA can be loaded in all the Flash Player Adobe plug-in browser to run in the same. Flex is through the Java or. Net and other non Flash way to explain the.Mxml file, and generate the corresponding.Swf file. Component of Flex and component of flash is very similar, but has improved. Flex at first as a J2EE (Java 2 Platform, Edition Enterprise), an application is released. FLASH applications can also be compiled by running the MXML (Flex markup language) and ActionScript. Currently the latest version of the FLEX has been to support the programmer to create a static file, the static file to use the way to compile the deployment, so that programmers more convenient to develop RIA applications [3]. 

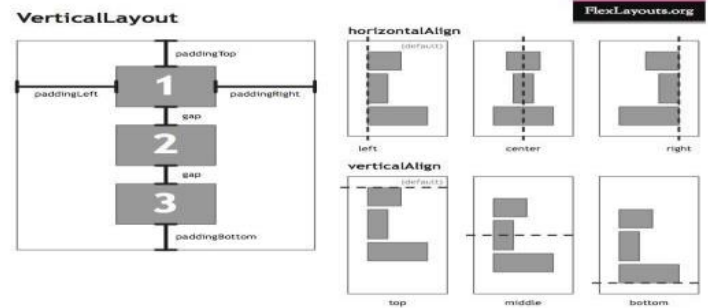

Fig. 1. Schematic diagram of flex technology structure

\subsection{Introduction and Analysis of J2EE Technology}

Java is a simple, object-oriented, robust, secure, interpreted, and platform independent, multi thread, dynamic language. Its structure is divided into three parts, the grammar, the running environment and the rich API. Object oriented is the most important characteristic of Java, which supports the object oriented characteristics such as inheritance, overloading, polymorphism and so on. Has been, people are beset with multi language universal platform, Java language by being compiled into middleware similar binary sequence, the can be in arbitrary a fitted with running on the hardware platform of the Java virtual machine, this way makes Java is widely used in Internet related equipment.

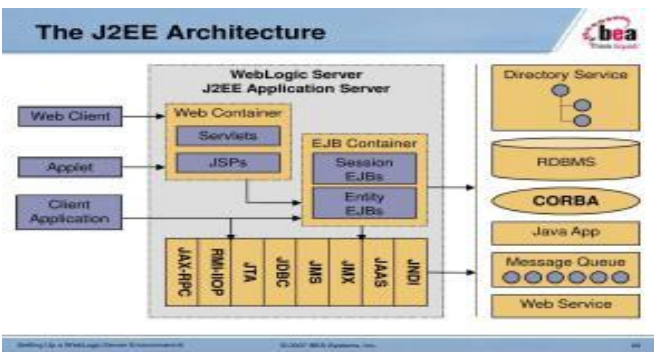

Fig. 2. Schematic diagram of J2EE technology

\subsection{Introduction and Analysis of Struts Technology}

Struts technology is one of the mainstream technologies in the current web development framework SSH, through the integration of traditional WebWork technology and Struts technology, the formation of the Struts2 technology framework. Because of its can make business logic and API Servlet isolation, Struts2 technology is widely used in Internet related services, also known as network system products [4]. 


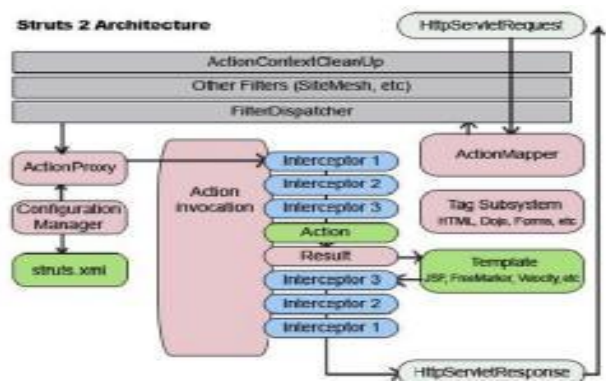

Fig. 3. Schematic diagram of struts technology

\section{System Design and Implementation}

Body shape analysis system to deal with a large number of data information, including the user's personal information and measurement information, etc.. This requires timely and effective storage and management of these data, to maintain the independence and integrity of the program and data, and to avoid redundant data. So the database is the foundation of the body shape analysis system. We designed the human body measurement database according to the needs of the data type of the 3D human body scanner and the data type of the garment manufacturing enterprise. The human body measurement database mainly contains the system user information, the measured body social attribute information, the measured body human body measurement information, the scanner information and so on various data. Security is one of the important aspects in body size analysis system. Because the measured data of the measured body is related to the privacy of the individual, it is necessary to manage the user and the data of the system. User rights refer to the user's user interface, including the right to add, modify, delete, Cha Xunquan, statistical analysis, data download rights, report generation right, confidential data view, etc.. Different levels of users with different permissions, such as low-level users only data add in, high level user is statistical analysis of rights, data download, report generation rights, confidentiality of data view of the kinds of rights. Good authority to ensure the security of the system and the human body measurement data. System users can according to the measured before scanning fill in the "Enclave user personal information table", will be measured the personal information input anthropometric database. The user's input process is composed of three user views, they are input request view, input failed view and input successful view. At the same time, it also relates to an input controller and input data model. User input business logic and data logic are implemented by means of a session component responsible for handling the business logic and a physical component responsible for processing the data logic. The session component is responsible for checking whether the user's input data is correct, the entity component is responsible for accessing the database, and returns the relevant data [5].

In order to make the garment production enterprises to better understand the target customer groups body feature, we in shape analysis system design of the statistical analysis module, garment production enterprises can according to occupation, age, region and other parameter properties to analyze the Somatotype Characteristics of the different groups. The results of statistical analysis can guide the production of clothing, and meet the needs of the production of eMTM. The statistical analysis module mainly has the mean analysis, the 
interval analysis and the percentage analysis these three kinds of analysis models. In the human body shape analysis system, we choose the clustering algorithm as the method of body classification. In order to make clothing designers from different angles on the human body size classification, we chose the K-means method and the method of identification of the two methods. At the same time, in order to make the shape classification results can better reflect the target population size characteristics, we from the anthropometric database selected measured body height, neck circumference, waist circumference, chest circumference, hip circumference as a clustering analysis of characteristic variables. Standard of our original shapes simply uses the chest and waist of the two parameters to determine the human body, and we choose the five anthropometric parameters can better reflect the human body characteristics, the shape analysis results with higher scientificity and accuracy, so as to satisfy the eMTM production on the size of the classification results of accuracy requirements.

\section{Conclusion}

Body shape analysis system from the anthropometric database extracted from the measured body height, neck circumference, waist circumference, chest circumference, hip circumference as shape analysis characteristic variables. The classification method in Chinese traditional simply will shape difference as the basis for classification of thoracolumbar. Therefore the size analysis system is able to make a more accurate data analysis on the characteristics of the human body, in order to more accurately reflect the human body characteristics and better meet the clothing prototype design and the requirements of design. At the same time, the system can also provide a good support for the research and teaching of clothing design theory research and teaching. Creatively introducing the method of cluster analysis into the research field of human engineering. Garment production enterprises to use the cluster analysis method for the size of the target user groups from different angles, and in fashion design experts involved in the analysis of shape classification results and determine the characteristics of each type. So the garment production enterprise can customize the clothing according to the different shape characteristic of the target user group, which greatly improves the market reaction ability of the garment production enterprise, and also meets the requirement of the user to the garment fit.

\section{References}

[1] Li Zhao. Talk about the human body effect on the left chest structure of. Textile industry and technology. No. 01. (2011)

[2] Li Haitao. Analysis on the influence of body size on garment making board. Tianjin Textile Science and technology. No. 01. (2012)

[3] listed clothing shall be marked with a new symbol of human body size. Sichuan textile technology. No. 04. (2014)

[4] He Huanhuan. A new logo. fiber standard and test. No. 11. (2013)

[5] Qian Xiaonong, Yin Bing. Research on human body segmentation and recognition based on 3D human body measurement. Journal of textile science. No. 02. (2011) 